\title{
Diferencias en el sentimiento de soledad entre adultos institucionalizados y no institucionalizados
}

\author{
Differences in the Feeling of Loneliness Among Institutionalized and \\ Non-institutionalized Elders
}

\section{Diferencia no sentimento de solidão entre adultos institucionalizados e não institucionalizados}

\author{
Ángela Quintero-Echeverri', María Mercedes Villamil-Gallego², Eucaris Henao-Villaª, Jairo León Cardona-Jiménez \\ 1 Psicóloga. Docente-investigadora Corporación Universitaria Remington. Medellín, Colombia. \\ 2 Trabajadora social. Magíster en Filosofía. Docente-investigadora Corporación Universitaria Remington. Medellín, \\ Colombia. \\ $3 \quad$ Psicóloga. Docente-investigadora Corporación Universitaria Remington. Medellín, Colombia. \\ 4 Nutricionista dietista, especialista en Educación para la Salud, magíster en Epidemiología. Docente-investigador \\ Corporación Universitaria Remington. Medellín, Colombia. Jairo.cardona@uniremington.edu.co \\ Grupo de Investigación en Salud del Adulto Mayor (GISAM), Programa de Medicina, Facultad de Ciencias de la Salud, \\ Corporación Universitaria Remington.
}

Recibido: 18/04/2017. Aprobado: 22/05/2018. Publicado: 30/04/2018

Quintero-Echeverri Á, Villamil-Gallego MM, Henao-Villa E, Cardona-Jiménez JL. Diferencias en el sentimiento de soledad entre adultos institucionalizados y no institucionalizados. Rev. Fac. Nac. Salud Pública. 2018;36(2):49-57. DoI:10.17533/udea.rfnsp. v36n2a07

\section{Resumen}

Objetivo: Comparar los niveles de soledad entre la población adulta institucionalizada y la no institucionalizada. Metodología: Investigación exploratoria de corte. Se midió el nivel de soledad reportada, usando una escala previamente validada (ESTE) en un grupo de adultos no institucionalizados, conformado por 869 mayores de 55 años, asistentes a los grupos de la tercera edad de las diferentes comunas de Medellín, captados mediante un muestreo aleatorio por conglomerados; asimismo, se aplicó en un grupo de adultos institucionalizados de 55 y más años de edad, residentes en centros gerontológicos públicos y privados, para un total de 379 adultos. Se analizaron las variables sociodemográficas: género, estado civil, escolaridad y edad, usando las pruebas U de Mann-Whitney y Kruskal-
Wallis, con una significancia de 0,05. Resultados: Se observó mayor puntaje de soledad familiar, social y conyugal en los adultos institucionalizados. La crisis de adaptación fue similar en ambos grupos. El puntaje de soledad fue significativamente diferente en los adultos no institucionalizados en las variables de estado civil, escolaridad y edad, mientras que en los institucionalizados solo se observó diferencias con el estado civil. El puntaje total de soledad fue mayor en quienes no tenían pareja y estaban institucionalizados. Conclusiones: El sentimiento de soledad fue mayor en adultos institucionalizados. La variable estado civil estuvo asociada con la soledad en ambos grupos.

-Palabras clave: adulto mayor, soledad, internado y residencia, condiciones sociales, institucionalización, envejecimiento. 


\begin{abstract}
Objective: to compare levels of loneliness between institutionalized and non-institutionalized populations. Methodology: exploratory research to measure the level of loneliness using a previously validated scale (ESTE) in a group of non-institutionalized elders made up of 869 people over the age of 55 who attended elderly groups at one of Medellin's neighborhoods, selected by random cluster sampling. The scale was also applied to a group of 379 institutionalized elders over the age of 55 residing at public or private gerontological centers. The sociodemographic variables analyzed were: gender, marital status, education and age. $U$ tests by Mann-Whitney and Kruskal-Wallis, with significance of 0,05 were used for this research. Results: a higher score in family, social and marital
\end{abstract}

loneliness was observed in institutionalized elders. The adaptation crisis was equal in both groups. The loneliness score of non-institutionalized adults was significantly different in the variables of marital status, education and age. Meanwhile, for the institutionalized adults, differences were only observed in the marital status variable. The total loneliness score was higher in institutionalized elders without a sentimental partner. Conclusions: the feeling of loneliness was overall higher in institutionalized elders. The marital status variable was associated with loneliness in both groups.

-Key words: elders, loneliness, institutionalized and residence, social conditions, institutionalization, old age.

\section{Resumo}

Objetivo: comparar os níveis de solidão entre a população institucionalizada e a não institucionalizada. Metodologia: pesquisa exploratória de corte. Foi possível medir a solidão, com uma escala previamente validada (ESTE), em um grupo de adultos não institucionalizados, conformado por 869 pessoas com mais de 55 anos, participantes dos grupos da terceira idade de várias comunas de Medellín, captados através de amostragem aleatória por conglomerados. Da mesma maneira, aplicou-se em um grupo de adultos institucionalizados de 55 ou mais anos de idade, residentes de centros gerontológicos públicos e particulares, para um total de 379 adultos. Analisaram-se variáveis sociodemográficas: género, estado civil, educação e idade, mediante provas U de Mann-Whitney e Kruskal-Wallis, com significância de 0,05. Resultados: observaram-se mais pontos de solidão familiar, social e conjugal nos idosos institucionalizados. A crise de adaptação foi similar em ambos os grupos. Os pontos de solidão foram significativamente diferentes nos adultos não institucionalizados nas variáveis de estado civil, educação e idade. Enquanto aos institucionalizados, só observou-se diferença no estado civil. O total de pontos de solidão foi mais alto nas pessoas sem companheiros sentimentais e estavam institucionalizadas. Conclusões: o sentimento de solidão foi mais alto em adultos institucionalizados. A variável de estado civil esteve associada com a solidão em ambos os grupos.

--------Palavras-chave: adulto idoso, solidão, internado e residência, condições sociais, institucionalização, velhice.

\section{Introducción}

Profundizar en la realidad de nuestros adultos mayores y sus vivencias constituye no solo una necesidad, sino también un desafío, dado el aumento de la esperanza de vida y el índice poblacional, pues implica la búsqueda de nuevos elementos que les permitan a nuestros mayores vivir en condiciones dignas y gratificantes.

A nivel mundial, se presenta un incremento del número de adultos mayores. En un estudio realizado por la Comisión Económica para América Latina y el Caribe (CEPAL) [1], se señala que, en todos los países de la región de América Latina y el Caribe, aumentará la proporción de personas de 60 años y más; este crecimiento será mayor que el de otros grupos etarios y será entre tres y cinco veces mayor que la población total en los períodos 2000-2025 y 2025-2050, respectivamente. En Colombia, el censo de 2005 informa que hay 2612508 adultos mayores de 65 años, correspondientes al 6,5\% del total; se espera que para el 2050, más del $20 \%$ (calculados en 72 millones) de los pobladores esté por encima de 60 años [2].

Debido a este incremento, la institucionalización, es decir, la internación en centros gerontológicos o geriátricos (asilos), toma cada vez más relevancia y se convierte en una necesidad sociofamiliar [3]. En los países desarrollados crece el porcentaje de adultos mayores institucionalizados, lo que implica que aumentaron los más ancianos, aquellos con escaso apoyo social y los más dependientes [4].

Anteriormente, la familia estaba compuesta por varias generaciones y los ancianos tenían un rol fundamental que cumplir, como ejes del grupo familiar; debido al surgimiento de una nueva estructura familiar, más reducida, se ha incrementado la institucionalización [5]. Un porcentaje alto de adultos mayores perciben el ingreso en una institución como una señal evidente de abandono por parte de la familia y la mayoría de ellos no fueron consultados al respecto [6]. 
En un estudio efectuado en Centros de Bienestar de adultos mayores de Medellín se encontró que el principal motivo que causó la institucionalización fue la soledad (el 23 \% vivía solo); desafortunadamente, este sentimiento permanece en ellos, debido, en parte, por el "poco contacto con las familias y la mediana satisfacción con amigos y la misma institución” [7].

En otro estudio realizado en Santiago de Cuba, en adultos institucionalizados y semiinstitucionalizados, el 35,5 y el $52,8 \%$, respectivamente, reportaron tendencia a la soledad [8]. En el distrito de Bragança (Portugal), al referirse a la depresión, las variables de mayor peso para explicarla fueron la soledad, seguida por una menor importancia dada a las actividades del tiempo libre y ser del género femenino [9]. En Chile, en una investigación con adultos institucionalizados, se halló que ellos sienten soledad, desesperanza o confusión en su vida: 33,7 \% casi a diario y 21,2\% de 1 a 3 veces por semana [10].

Molina, Meléndez y Navarro [11] compararon el bienestar y la calidad de vida en ancianos institucionalizados y no institucionalizados, encontrando que son superiores en los últimos, quienes además obtienen el porcentaje más alto en la percepción de bienestar desde el apoyo familiar. En general, los factores propios del bienestar subjetivo mostraron diferencias, siendo las puntuaciones de los ancianos institucionalizados inferiores.

En Costa Rica, en una muestra de adultos mayores institucionalizados, los resultados evidencian que la internación geriátrica acelera el deterioro físico y psicológico, y aumenta el grado de dependencia, debido a sentimientos de soledad por encontrarse separados de su núcleo familiar, mostrándose apáticos, poco participativos en las actividades programadas, con mayores factores de riesgo como sedentarismo y aislamiento [12].

Tijhuis et al. [13] mencionan, en su investigación, que el aumento de la soledad experimentada por hombres muy viejos se ve influida por la pérdida de la pareja, mudarse a un hogar gerontológico y no sentirse saludable.

Así como la institucionalización se relaciona con la soledad, este sentimiento se asocia con variables sociodemográficas, como género, estado civil, edad, estatus socioeconómico $[14,15]$ y nivel educativo $[15,16]$. El ser mujer y el estado civil tienen una fuerte relación con la soledad; los no casados son más propensos a sentirse solos; los viudos, separados y divorciados expresan un mayor sentimiento de soledad que los casados [17-19]. También la edad está asociada con la soledad: a mayor edad, es mayor la soledad, y los adultos mayores de 80 años son los más vulnerables a la soledad [19]; Almeida y Quintao [20] encontraron que la soledad, la depresión y la ideación suicida son más recurrentes en las personas que tienen más edad. La educación y el ingreso se asocian negativamente con la soledad, puesto que a mayor nivel educativo e ingresos, es menor la soledad; adicionalmente, dichos factores benefician las relaciones sociales en cantidad y calidad, siendo esta última la más relevante [21]. Pinquart y Sörensen [15], en su estudio, indicaron que la soledad en adultos mayores tiene relación con la calidad y la cantidad del contacto; prima la calidad.

Para Yuste Rosell [22], el significado de la institucionalización o el asilo, ya no es propio de personas que son abandonadas, sino que puede conducir a las personas a experiencias gratificantes, como la adquisición de un mayor número de amistades.

Por tanto, identificando que en la bibliografía consultada existe una fuerte inclinación a puntualizar las dificultades en la institucionalización, este estudio pretendió comparar los niveles de soledad entre la población adulta institucionalizada residente en centros gerontológicos de la ciudad de Medellín, tanto vinculados al municipio de Medellín, como los de carácter privado, y los adultos no institucionalizados que asistían a los grupos de la tercera edad del municipio de Medellín, entre los años 2011 y 2013.

Se contó con el listado de grupos de la tercera edad del municipio de Medellín, en el que aparecían las variables sociodemográficas: género, estado civil, escolaridad y edad, con el fin de ampliar el conocimiento de la población adulta en riesgo de soledad y poder facilitar la intervención temprana.

\section{Metodología}

Investigación exploratoria de corte transversal, que describe las diferencias en las variables de género, estado civil, escolaridad y edad, en la soledad reportada entre los adultos mayores institucionalizados y los no institucionalizados

Para medir la soledad, se utilizó la escala ESTE [17], basada en las escalas: UCLA, SELSA, ESLI, y la escala de satisfacción vital de Filadelfia, la cual se compone de cuatro ítems: soledad familiar, que está ligada con las relaciones familiares; soledad conyugal, que tiene que ver con la relación de pareja; soledad social, que hace referencia al contacto con el otro, y la crisis de adaptación, que se relaciona con la percepción del individuo frente a sí y la forma como enfrenta situaciones, como el envejecimiento. Esta escala fue validada en Colombia, obteniendo un Alfa de Cronbach de 0,90 [23].

Inicialmente se contaba con los datos de los adultos no institucionalizados; luego se tomaron los datos de los adultos institucionalizados, población que por su misma situación registró niveles de soledad muy altos; pero como el interés de los investigadores era registrar las variables que dieran luces sobre la diferencia entre estos sentimientos de soledad entre ambos grupos, por ello se analizaron en conjunto ambas bases de datos. 
Adultos no institucionalizados: se utilizaron los datos obtenidos en el trabajo de investigación "El sentimiento de soledad en adultos" [19], realizado por los autores de este mismo estudio en la ciudad de Medellín en el año 2011; se efectuó el análisis en 869 de ellos.

Se contó con el listado de grupos de la tercera edad del municipio de Medellín y con 25408 adultos asistentes registrados. La muestra se calculó de acuerdo con el número de personas que asistían a estos grupos por comuna, y de manera aleatoria se ubicaron los grupos, mediante un muestreo aleatorio por conglomerados (error estimado $3,3 \%$ ) hasta completar el número requerido para cada comuna.

Para la aplicación del instrumento se ubicaron los coordinadores de grupos de la tercera edad de las diferentes comunas de Medellín, se explicó el instrumento escala ESTE para medir la soledad y, posteriormente, se aplicó a los adultos mayores de 55 años que desearon participar de forma voluntaria en el estudio, con capacidad mental para responder la encuesta y asistentes a los diversos grupos.

Adultos institucionalizados: constituida por los adultos de 55 y más años que desearon participar en el estudio y con capacidad mental (según informe de la institución) para responder la encuesta, residentes en centros gerontológicos de la ciudad de Medellín, tanto vinculados al municipio de Medellín, como los de carácter privado, para un total de 379. Para la obtención de la muestra se contó con el listado de centros gerontológicos brindado por la Administración Municipal; se ubicó al director y se procedió en la aplicación de la encuesta.

Se sistematizó la información con los programas Epi Info $^{\circledR}$ 2005, SPSS $^{\circledR}$, versión 21, y Excel ${ }^{\circledR}$. Para el análisis se emplearon frecuencias absolutas, proporciones, promedios y medianas. Como estadísticos de prueba para las variables dependientes continuas, dada la no distribución normal de los resultados, se usaron las pruebas U de Mann-Whitney y Kruskal-Wallis, con una significancia de 0,05.

En el proceso de la recolección de la información, cada uno de los participantes firmó un documento donde se especificó el manejo confidencial y anónimo de los colaboradores, y la autorización para realizar la entrevista (consentimiento informado). Se tienen en cuenta los lineamientos trazados por la Resolución 8430 de 1993 del Ministerio de Salud, que precisa el manejo de las investigaciones donde participan seres humanos; el estudio se considera sin riesgo [24].

\section{Resultados}

A continuación se presentan los resultados de comparar los niveles de soledad en los adultos mayores institucionalizados y no institucionalizados.

Se observaron diferencias significativas en relación con el género entre las personas mayores instituciona- lizadas y las que no lo estaban, siendo el porcentaje de mujeres mayor en el grupo de personas no institucionalizadas $(86,8 \%)$. De acuerdo con la edad, una alta proporción de adultos institucionalizados son mayores de 75 años (54,6\%); en los que no lo están, el grupo predominante es menor de $65(44,4 \%)$.

Referente al nivel educativo, aquellos que no realizaron estudios representan un mayor porcentaje en los adultos institucionalizados; la primaria completa e incompleta es levemente mayor en los adultos no internos, al igual que la educación superior.

Con relación al estado civil, predominan aquellos que no tienen pareja, en especial en los institucionalizados, siendo, en estos, mayor la proporción de separados y viudos. Todas estas diferencias fueron significativas (véase tabla 1).

Al comparar el tipo de soledad entre los adultos institucionalizados y aquellos que no lo están, se observa una mayor soledad de todos los tipos en aquellos que se encuentran institucionalizados, a excepción de la crisis de adaptación, donde no se aprecian diferencias estadísticas significativas entre ambos (véase tabla 2).

La soledad en los géneros es similar en los institucionalizados y en los no que no lo están (diferencias no significativas); sin embargo, el total de soledad es menor en hombres y mujeres no institucionalizados, siendo estas diferencias estadísticamente significativas (véase figura 1).

El estado civil muestra diferencias en el total de soledad, tanto en los institucionalizados, como en aquellos que no lo están.

Los adultos con pareja, en ambos grupos, tienen menor nivel de soledad (diferencias no significativas entre ellos). El total de soledad se incrementa en quienes no tienen pareja y están institucionalizados (diferencias significativas) (véase figura 2).

La escolaridad está asociada estadísticamente con los puntajes de soledad total, solo en los no institucionalizados. En los que residen en instituciones, el nivel de soledad aumenta a medida que se incrementa el nivel de estudios, hasta llegar a los que alcanzaron estudios superiores, donde desciende levemente. Los de menor puntaje en el total de soledad fueron los de más bajo nivel educativo. Estas diferencias no fueron significativas ( $p=0,085$ ) (véase figura 3 ).

En los adultos no institucionalizados se observa un constante descenso del nivel de soledad a medida que se incrementa el nivel de escolaridad alcanzado $(p<0,00)$.

Al observar por nivel educativo, aquellos que no realizaron ningún grado no presentan diferencias significativas entre los institucionalizados y los que no lo están. En aquellos que cursaron la primaria y la secundaria completa e incompleta se incrementa de manera significativa en los institucionalizados, mientras desciende en los no institucionalizados. $(p<0,00)$. No se encontraron diferencias significativas entre quienes cursaron algún grado de educación superior $(p<0,107)$ (véase figura 3 ). 
Tabla 1. Variables sociodemográficas. Comparación entre adultos institucionalizados y no institucionalizados.

\begin{tabular}{|c|c|c|c|c|c|c|}
\hline & \multirow{2}{*}{ Variables } & \multicolumn{2}{|c|}{ No institucionalizados } & \multicolumn{2}{|c|}{ Institucionalizados } & \multirow[t]{2}{*}{$p$} \\
\hline & & $\mathrm{n}=869$ & $\%$ & $\mathrm{n}=379$ & $\%$ & \\
\hline \multirow{2}{*}{ Género } & Femenino & 754 & 86,8 & 224 & 59,1 & \multirow{2}{*}{0,000} \\
\hline & Masculino & 115 & 13,2 & 155 & 40,9 & \\
\hline \multirow{3}{*}{$\begin{array}{l}\text { Edad (en } \\
\text { años) }\end{array}$} & Menores de 65 & 386 & 44,4 & 45 & 11,9 & \multirow{3}{*}{0,000} \\
\hline & $65-75$ & 338 & 38,9 & 127 & 33,5 & \\
\hline & Mayores de 75 & 145 & 16,7 & 207 & 54,6 & \\
\hline \multirow{4}{*}{$\begin{array}{l}\text { Nivel } \\
\text { educativo }\end{array}$} & Ninguno & 84 & 9,7 & 73 & 19,4 & \multirow{4}{*}{0,000} \\
\hline & Primaria completa e incompleta & 575 & 66,2 & 220 & 58,4 & \\
\hline & Secundaria completa e incompleta & 171 & 19,7 & 74 & 19,6 & \\
\hline & Superior completa e incompleta & 39 & 4,5 & 10 & 2,7 & \\
\hline \multirow{4}{*}{$\begin{array}{l}\text { Estado } \\
\text { civil }\end{array}$} & Con pareja & 394 & 45,3 & 35 & 9,3 & \multirow{4}{*}{0,000} \\
\hline & Separado/a & 66 & 7,6 & 56 & 14,9 & \\
\hline & Soltero/a & 119 & 13,7 & 168 & 44,6 & \\
\hline & Viudo/a & 290 & 33,4 & 118 & 31,3 & \\
\hline
\end{tabular}

Prueba chi-cuadrado de Pearson.

Tabla 2. Diferencias en los puntajes según tipos de soledad entre los adultos institucionalizados y no institucionalizados.

\begin{tabular}{lcccc}
\hline \multirow{2}{*}{ Tipo de soledad } & \multicolumn{1}{c}{ Institucionalizados } & No institucionalizados & Rango & \multirow{2}{*}{$\begin{array}{c}\text { Prueba U de } \\
\text { Mann-Whitney }\end{array}$} \\
\cline { 2 - 4 } & $\ddot{X}(\mathrm{Me})$ & $\ddot{X}(\mathrm{Me})$ & Mín.-máx. & \\
\hline Familiar & $11,8(12)$ & $6,5(4)$ & $4-20$ & 0,000 \\
Conyugal & $23,2(25)$ & $16,5(21)$ & $5-25$ & 0,000 \\
Social & $20,3(19)$ & $14,9(12)$ & $8-40$ & 0,000 \\
Adaptación & $29,9(26)$ & $29,7(28)$ & $13-65$ & 0,520 \\
\hline Total & $85,2(83)$ & $67,6(65)$ & $30-150$ & 0,000 \\
\hline
\end{tabular}

Prueba de rango de Wilcoxon.

Sig. $p=0,000$.

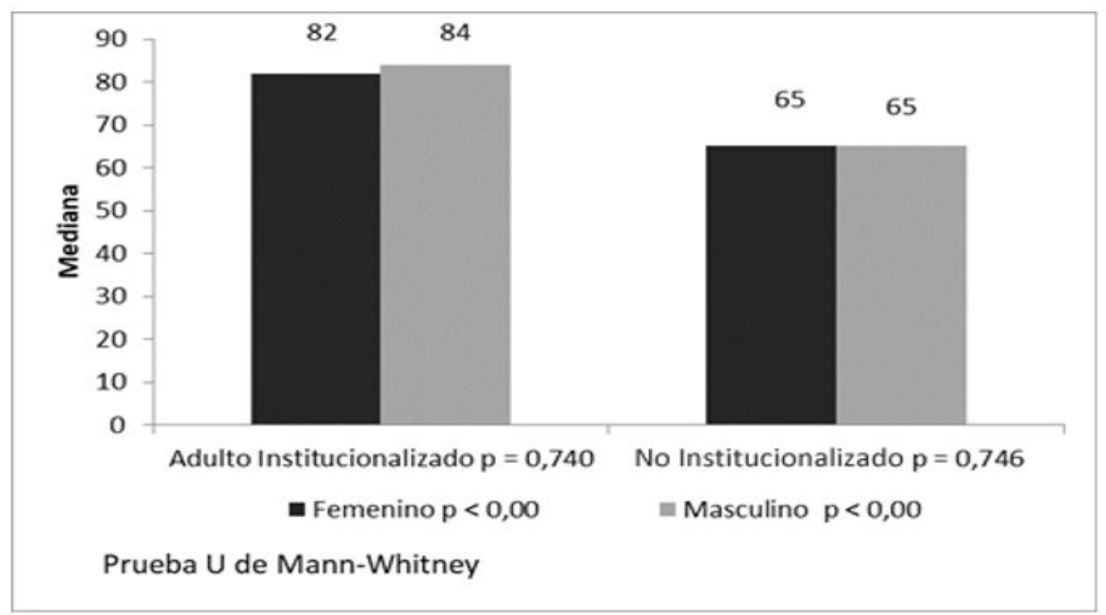

Figura 1. Diferencias en puntajes (Mediana) de soledad entre adultos institucionalizados y no institucionalizados según género. 


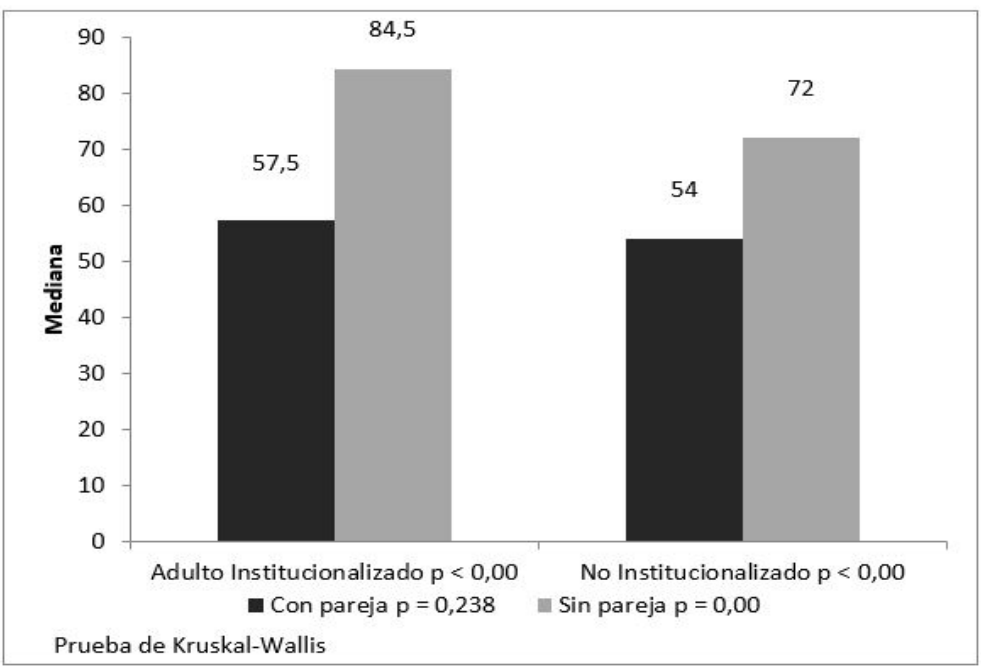

Figura 2. Diferencias en puntajes (Mediana) de soledad entre adultos institucionalizados y no institucionalizados según estado civil.

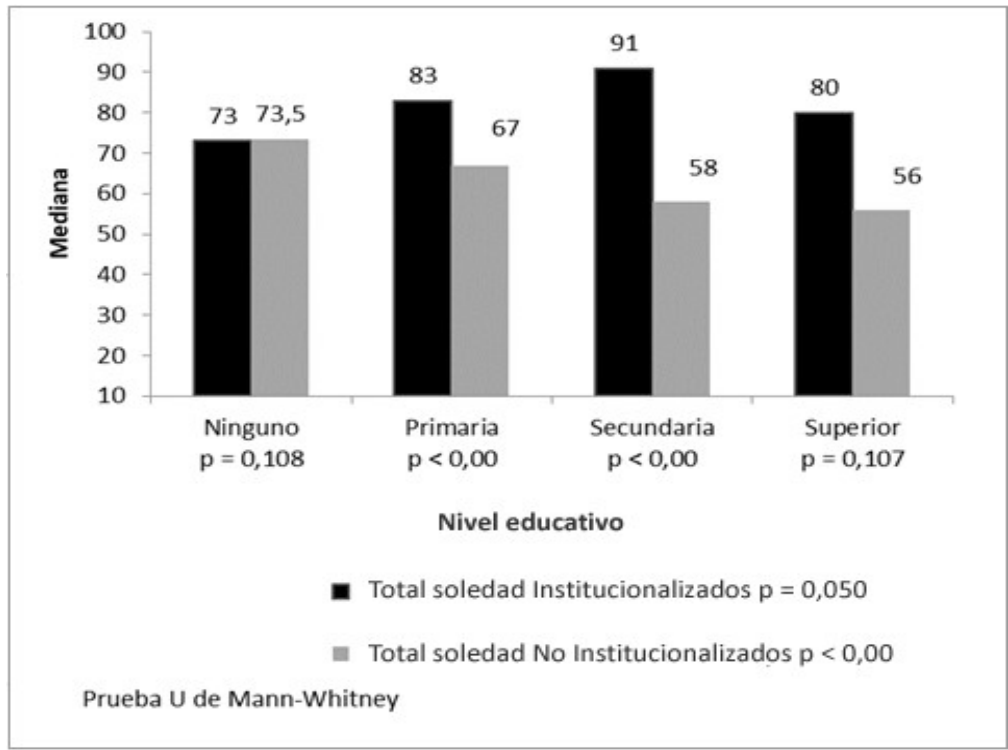

Figura 3. Diferencias en puntajes (Mediana) de soledad entre adultos institucionalizados y no institucionalizados según escolaridad

La soledad se incrementa de manera significativa en los adultos no institucionalizados, a medida que aumenta la edad.

En los adultos institucionalizados crece levemente la soledad a medida que aumenta la edad, sin presentarse diferencias significativas $(p=0,861)$; en el mismo sentido, se incrementa la soledad en los no institucionalizados, pero con diferencias más amplias, alcanzando a tener diferencias significativas entre los diversos grupos de edad $(p<0,000)$. Al comparar los diferentes grupos de edad según las personas residentes en instituciones, se observa que el nivel de soledad es mayor en los institucionalizados $(p<0,00)$ (véase figura 4).

\section{Discusión}

Los resultados muestran mayor soledad en los adultos institucionalizados en sus diferentes tipos, lo cual concuerda con lo afirmado por distintos autores, como Molina, Meléndez y Navarro [11], Villalobos [12] y Tijhuis et al. [13], quienes indican que la institucionalización puede favorecer el sentimiento de soledad en los adultos mayores.

La soledad familiar es experimentada por los adultos mayores institucionalizados en un mayor nivel $(\mathrm{Me}=12$, de un máximo de 20), en comparación con los que no lo están, pues el nivel es mínimo $(\mathrm{Me}=4$ 


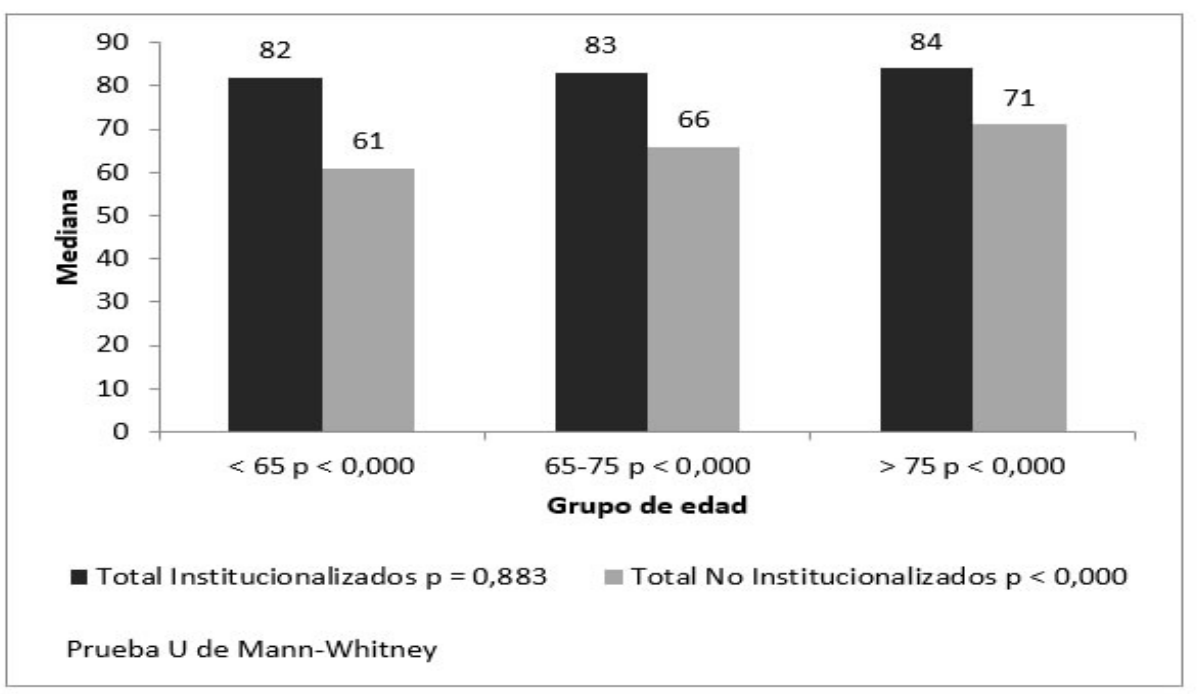

Figura 4. Diferencias en puntajes (Mediana) de soledad entre adultos institucionalizados y no institucionalizados según edad.

de un máximo de 20). Este aspecto se puede relacionar, por otra parte, con el menor tiempo que tienen los familiares para visitar a su pariente, dadas las diversas actividades que realizan como el trabajo, el estudio, el cuidado de los hijos, la vida social.

En el mismo sentido, los resultados concuerdan con el trabajo de Molina et al. [11]: la soledad es menor en los adultos mayores no institucionalizados, quienes además obtienen el porcentaje más alto en la percepción de bienestar desde el apoyo familiar.

El abandono y el desinterés de la familia están presentes en el pensamiento del anciano [6]; anteriormente era frecuente pertenecer a una red extensa y el adulto mayor tenía un lugar, un rol y era dicho núcleo quien le ofrecía los cuidados necesarios; en la actualidad, la familia no tiene en cuenta en su estructura al anciano y le niega su espacio habitual, y lo relega a un papel pasivo, generando en él baja autoestima y sufrimiento por el desamparo $[5,6]$. Villalobos [12] expresa que la institucionalización precipita el detrimento del adulto, debido a sentimientos de soledad generados por la separación de su grupo familiar.

Por su parte, la soledad conyugal se refleja en mayor medida en los adultos institucionalizados, lo cual puede explicarse por la ausencia de pareja en el $91 \%$ de ellos, que lleva incluso a que muchos familiares opten por internar al adulto para que se relacione con otras personas y no sienta tanto la ausencia de su pareja; sin embargo, como lo refieren Cardona et al. [7], la primera causa de institucionalización es la soledad, pero esta continúa, aun después del ingreso al centro gerontológico.

La soledad social es percibida en un mayor nivel en los adultos mayores que viven en instituciones. Esto se puede explicar por la separación de su familia, amigos y vecinos. La mayoría de los adultos mayores ingresan a la institución porque estaban solos, pero esta soledad permanece [7], puesto que las relaciones que encuentran allí, aunque son muchas, no son necesariamente de calidad y de su entera satisfacción [11].

El apoyo social es importante dentro del concepto de envejecimiento óptimo e influye de manera significativa sobre la calidad de vida del anciano [13]. Yuste [22] refiere que el internamiento puede propiciar un nuevo círculo de amistades, pero es claro que no se da en los centros gerontológicos del estudio; y según Hawkley et al. [21], y Pinquart y Sörensen [15], es más importante estar satisfecho con las relaciones de la red personal, independiente del tamaño de esta. Asimismo, los adultos institucionalizados cuentan principalmente con los vínculos al interior de la institución, debido a que para salir por fuera de la misma, presentan diversas dificultades, como los permisos, el estado de salud y la falta de recursos.

Respecto a la crisis de adaptación, la cual tiene que ver con la percepción del individuo frente a sí mismo y la forma como enfrenta situaciones como el envejecimiento, es similar tanto en adultos no institucionalizados, como en los que no lo están, lo cual implica que hay, en el adulto mayor, factores personales que son independientes de la situación externa, aspectos que lo hacen más vulnerable y que son necesarios fortalecer.

En relación con el género, la soledad es mayor en hombres y mujeres institucionalizados, sin diferencias significativas entre ellos; pero al compararlos con los adultos de ambos géneros no institucionalizados, sí se encuentran estas diferencias importantes, lo que representa que, para los dos géneros, en la institucionalización se incrementa la soledad, debido a factores independientes. 
En el estudio de Villamil et al. [19], en adultos no institucionalizados que asisten a centros abiertos de tercera edad del municipio de Medellín, tampoco se hallaron diferencias significativas en el nivel total de soledad respecto al género, pero sí al interior de sus tipos; los hombres reportaron mayor soledad familiar y crisis de adaptación, mientras las mujeres enunciaron mayor soledad conyugal, y al realizar las sumatorias para obtener el total de soledad, estas se equiparan. En tal sentido, hay controversia en cuanto a cuál género tiene mayor grado de soledad.

En referencia al estado civil, diversos autores lo asocian con la soledad $[14,15,18,19]$. Resalta la no existencia de diferencia significativa en el sentimiento de soledad entre los adultos institucionalizados y aquellos que no lo están, en quienes cuentan con pareja en la actualidad; el contar con pareja se convierte en un factor protector contra la soledad, independiente del medio externo. En las personas que no tienen pareja, la soledad se incrementa de manera especial en aquellos que residen en instituciones, donde el 44,6\% son solteros y cuentan con pareja actualmente el 9,3\%, contra el 13,7 y 45,3\%, respectivamente, en quienes no están internos. Parece que tienen mayores posibilidades de combatir la soledad entre quienes no tienen pareja, aquellos que no están institucionalizados; además, la red social frecuentada brinda apoyo, mejorando el sentimiento de bienestar.

En cuanto a la escolaridad, se aprecia que el nivel de soledad se relaciona de manera inversa con el grado educativo en los adultos no institucionalizados, lo que concuerda con diversos estudios $[15,16,20]$; pero no se sigue el mismo esquema en los adultos institucionalizados, donde quienes experimentan menor soledad son los que tienen bajo nivel de escolaridad, lo que podría ser explicado por la relación entre la baja escolaridad y las privaciones económicas [20], al habitar el adulto en una institución donde se llenan sus necesidades básicas de manera satisfactoria, lo que no pasa en los no institucionalizados, quienes deben suplir dichas necesidades con sus recursos y que tienen estrecha relación con la escolaridad alcanzada.

Con respecto a la edad, se encontró mayor soledad entre los adultos institucionalizados. Adicionalmente, en ambos grupos se presenta que a medida que aumenta la edad, también se incrementa la soledad. Resultados semejantes son expresados por otros autores $[15,17,19]$. Con la edad se dan pérdidas en los recursos sociales y personales, las cuales pueden poner a los adultos mayores en riesgo de aislamiento y soledad, y pueden afectar de manera negativa su calidad de vida. Además, las pérdidas en cuanto al empeoramiento de la salud aumentan la probabilidad de la soledad [18]. Igualmente, el adulto mayor, a medida que avanza su edad, va perdiendo amigos y pueden incrementarse las dificultades para relacionarse con otros.
En resumen, se halló mayor soledad familiar, social y conyugal en los adultos institucionalizados. La crisis de adaptación es similar en los adultos institucionalizados y los que no lo están, lo cual muestra que existen, en ambos grupos, aspectos internos que son independientes de la condición externa.

Para los adultos no institucionalizados, las diferencias en las variables de estado civil, escolaridad y edad aparecen asociadas significativamente, mientras que en los institucionalizados, solo se da con el estado civil.

El sentimiento de soledad es mayor en los adultos institucionalizados. Los efectos de la vejez son adversos, más aún para el que vive en una institución. Tener que adaptarse a un nuevo espacio, con nuevas personas y nuevas reglas puede afectar su bienestar, puesto que el adulto, además de perder su independencia y su rol social, deja de tener un contacto cercano de vecinos, familiares y amigos. Por lo anterior, es recomendable, de acuerdo con las posibilidades, que el adulto mayor permanezca en el propio entorno y, en caso contrario, se hace necesario que las residencias geriátricas y gerontológicas desarrollen nuevas estrategias a nivel social y familiar, de tal forma que el adulto mayor no sea tan vulnerable a la soledad.

Se sugiere a las entidades que tienen a su cargo de manera permanente adultos mayores, que fortalezcan espacios de diálogo individuales y con familias, y a su vez incrementen espacios sociales en el diario vivir de la entidad, de manera que se pueda contrarrestar algunas dificultades que se presenten en la socialización con algunos adultos mayores.

Asimismo, es fundamental revisar y trabajar la crisis de adaptación en ambos grupos poblacionales, de modo que cada vez el individuo acepte de mejor forma su estado actual. Pero esto es una tarea que cobija no solo a los adultos mayores, sino también al resto de la población que llegará a ser adulta, y sigue mostrando la importancia de prepararse para llegar a esta edad.

\section{Limitaciones}

El estudio es de corte. Se tomó una sola medida para cada grupo, por lo tanto, los niveles de soledad fueron las encontradas en el momento de la aplicación de la encuesta, y las asociaciones estadísticas halladas deben ser observadas con precaución y verificadas con otro tipo de estudio.

La conformación de los grupos (institucionalizados y no) de antemano suponen un mayor nivel de soledad en los primeros, dadas por la forma como se estructuran estos grupos.

Para profundizar en el tema es necesario realizar un estudio cualitativo, donde a través de entrevistas a adultos mayores y diario de campo se profundice en las diferencias existentes.

\section{Agradecimientos}


El Grupo de Investigación en Salud del Adulto mayor (GISAM) agradece la colaboración de las directivas de la Corporación Universitaria Remington, a la Vicerrectoría de Investigaciones, a los directores y coordinadores de los centros gerontológicos y clubes de vida, y a todos los adultos mayores que participaron en el estudio.

\section{Fuentes de financiación}

Este proyecto, financiado por la Corporación Universitaria Remington, hace parte del proyecto "Investigación y desarrollo: soledad en el adulto no institucionalizado del municipio de Medellín”, con código: 4000000021-1 de 2014.

\section{Declaración de conflicto de intereses}

Los autores declaran no tener ninguno conflicto de intereses.

\section{Referencias}

1 Comisión Económica para América Latina y el Caribe (CEPAL). Las personas mayores en América latina y el Caribe: diagnóstico sobre la situación y las políticas. Conferencia regional intergubernamental sobre envejecimiento: hacia una estrategia regional de implementación para América Latina y el Caribe del Plan de Acción Internacional de Madrid sobre el Envejecimiento; 2003 nov. 19-21; Santiago de Chile; 2003.

2 Ministerio de la Protección Social. Fundación Saldarriaga Concha. Diagnóstico de los adultos mayores en Colombia. Diagnóstico preliminar sobre personas mayores, dependencia y servicios sociales en Colombia. Bogotá: mimeo; 2007.

3 Marín P, Guzmán J, Araya A. Adultos mayores institucionalizados en Chile: ¿Cómo saber cuántos son? Rev Méd Chile. 2004;132(7):832-8.

4 Hirschfeld M, Lindsey E. Community home-based care in resource-limited settings: A framework for action. wHO; 2002.

5 Cifuentes P. Adulto mayor institucionalizado. [Tesis para optar al grado académico de licenciada en Trabajo Social]. Santiago, Chile: Universidad Academia de Humanismo Cristiano Escuela de Trabajo Social; 2005.

6 Iglesias de Ussel J. La soledad en las personas mayores: influencias personales, familiares y sociales. Análisis cualitativo. Madrid: Instituto de Migraciones y Servicios Sociales (IMSERSO); 2001.

7 Cardona D, Estrada A, Chavarriaga L, Segura A, Ordoñez J, Osorio J. Apoyo social dignificante del adulto mayor institucionalizado. Rev Salud Pública. 2010;12(3):414-24.

8 Soria B, Bandera D, Merceron Y, Zamora M. Aspectos psicológicos y sociales más relevantes en ancianos institucionalizados. Rev Cubana Enfermer. 1999;15(3):207-12.

9 Alves S, Soares N. Depressão em idosos institucionalizados no distrito de Bragança. Revista de Enfermagem Referência. 2011;3(4):49-58
10 Sepúlveda C, Rivas E, Bustos L, Illesca M. Perfil socio-familiar en adultos mayores institucionalizados. Temuco, Padre las Casas y Nueva Imperial. Cienc. Enferm. 2010;16(3):49-61.

11 Molina C, Meléndez JC, Navarro E. Bienestar y calidad de vida en ancianos institucionalizados y no institucionalizados. Anales de Psicología. 2008;24(2):312-9.

12 Villalobos D. Problemas de salud que enfrenta la población de la tercera edad en la ciudad de Heredia. [Tesis de Magíster Scientiae]. UCR. San José, Costa Rica; 1989. Citado por: Mora M, Villalobos D, Araya G y Ozols A. Perspectiva subjetiva de la calidad de vida del adulto mayor, diferencias ligadas al género y a la práctica de la actividad físico recreativa. Revista MHSalud. 2004;1(1):1-12.

13 Tijhuis M, De Jong J, Feskens E, Kromhout D. Changes in and factors related to loneliness in older men. The Zutphen Elderly Study. Age Ageing 1999;28(5):491-5.

14 Theeke L. Predictors of loneliness in U.S. adults over age sixtyfive. Arch Psychiatr Nurs. 2009;23(5):387-96.

15 Pinquart M, Sörensen, S. Influences on loneliness in older adults: A meta-analysis. Basic Appl Soc Psych. 2001;23(4):245-66.

16 Bishop A, Martin P. The indirect influence of educational attainment on loneliness among unmarried older adults. Educational Gerontology. 2007;33(19):897-917.

17 Rubio R. La soledad en las personas mayores españolas [Internet]. Madrid: CSIC; 2003 [Consultado 12 de junio de 2011]. Disponible en: http:/www.imsersomayores.csic.es/documentos/ documentos/rubio-soledad-01.pdf

18 Demakakos P, Nunn S, Nazroo J. Loneliness, relative deprivation and life satisfaction. En: Banks J, Breeze E, Lessoff C, Nazroo J, editores. Retirement, health and relationships of the older population in England: The 2004 English longitudinal study of ageing. London: Institute for Fiscal Studies; 2006.

19 Villamil M, Quintero Á, Henao E, Cardona J. El sentimiento de soledad en adultos. Medicina U.P.B. 2013;32(1):9-19.

20 Almeida L, Quintao S. Depressão e ideação suicida em idosos institucionalizados e não institucionalizados em Portugal. Acta Med Port. 2012;25(6):350-8.

21 Hawkley L, Hughes M, Waite L, Masi C, Thisted R, Cacioppo J. From social structural factors to perceptions of relationship quality and loneliness: The Chicago Health, Aging, and Social Relations Study. J Gerontol B Psychol Sci Soc Sci. 2008;63(6):375-84.

22 Yuste N. La problemática social de los cambios poblacionales. En: Yuste Rossel N, Rubio Herrera R, Rico MA. Introducción a la psicogerontología. Madrid. Ediciones pirámide; 2004. pp. 89-94.

23 Cardona-Jiménez J, Quintero-Echeverri Á, Villamil-Gallego M, Henao-Villa E. Validación de la escala este para medir la soledad de la población adulta. Invest. Educ. Enfer. 2010;28(3):416-27. Disponible en: http://www.redalyc.org/articulo.oa?id=105215721012

24 Resolución número 8430 de octubre 4 de 1993, por la cual se establecen las normas científicas, técnicas y administrativas para la investigación en salud. Bogotá: Ministerio de Salud; 1993. 